\title{
Relevance of equilibrium in multifragmentation
}

\author{
Takuya Furutd \\ GANIL (DSM-CEA/IN2P3-CNRS), B.P.55027, F-14076 Caen, France \\ Akira Ono \\ Department of Physics, Tohoku University, Sendai 980-8578, Japan
}

\begin{abstract}
The relevance of equilibrium in a multifragmentation reaction of very central ${ }^{40} \mathrm{Ca}+{ }^{40} \mathrm{Ca}$ collisions at 35 $\mathrm{MeV} /$ nucleon is investigated by using simulations of antisymmetrized molecular dynamics (AMD). Two types of ensembles are compared. One is the reaction ensemble of the states at each reaction time $t$ in collision events simulated by AMD, and the other is the equilibrium ensemble prepared by solving the AMD equation of motion for a many-nucleon system confined in a container for a long time. The comparison of the ensembles is performed for the fragment charge distribution and the excitation energies. Our calculations show that there exists an equilibrium ensemble that well reproduces the reaction ensemble at each reaction time $t$ for the investigated period $80 \leq t \leq 300 \mathrm{fm} / c$. However, there are some other observables that show discrepancies between the reaction and equilibrium ensembles. These may be interpreted as dynamical effects in the reaction. The usual static equilibrium at each instant is not realized since any equilibrium ensemble with the same volume as that of the reaction system cannot reproduce the fragment observables.
\end{abstract}

PACS numbers: $25.70 . P q$

\section{INTRODUCTION}

In medium-energy heavy-ion collisions at around the Fermi energy, intermediate-mass fragments as well as a large number of light particles such as nucleons and alpha particles are copiously produced [1, 2, 3, 4, 5]. This phenomenon is called multifragmentation. It is a challenging problem to understand the complex but rich quantum many-body dynamics of multifragmentation. One of the purposes of studying heavy-ion collisions is to explore the properties of nuclear matter [6, 7]. This information is valuable not only for nuclear physics but also for astrophysical interests such as supernova explosions and the structure of neutron stars [8]. The nuclear matter is expected to be compressed in the initial stage of a collision and the created compressed matter then expands afterward. The study of heavy-ion collisions thus offers a possibility to probe the properties of nuclear matter in a wide range of density. Multifragmentation has been considered to occur in the expanding stage and to have some connection to the nuclear liquid-gas phase transition, the existence of which is speculated based on the resemblance between the equation of state of homogeneous nuclear matter and that of a van der Waals system. Intensive research has been carried out to find evidence of this phase transition in experimental data of multifragmentation. In some works it is claimed that indications have been obtained [5, 9, 10, 11, 12, 13, 14, 15]. However, they are not conclusive and much effort is still required.

One of the difficulties is that it is not straightforward to relate the experimental data of heavy-ion collisions with the statistical properties of nuclear matter unless the state variables such as the temperature are well defined in dynamical reactions. The typical reaction time scale of multifragmen-

*Present address: LPC Caen (CNRS-IN2P3/ENSICAEN et Université), F14050 Caen, France. tation reactions is the order of $100 \mathrm{fm} / c$, which may not be long enough for the system to reach equilibrium compared with the typical time scale of successive two-nucleon collisions (a few tens of $\mathrm{fm} / \mathrm{c}$ ). However, there are several reports that support the achievement of equilibrium. An example is the existence of several types of scaling laws that appear in experimental data (e.g. Fisher's scaling [13] and isoscaling $[16,17])$, which may be understood if the system has reached equilibrium. Another example is the reasonable reproduction of the fragment mass (charge) distribution by statistical models for some multifragmentation reactions [18, 19, 20, 21, 22]. However, the achievement of equilibrium in multifragmentation reactions is still a controversial issue. One of the difficulties is that the information obtained directly from experiments is that of the very last stage of the reactions. Even if the system reaches equilibrium, the system undergoes the sequential decay process that distorts the information at the stage of the equilibrium before the fragments are finally detected in experiments. Another difficulty is that even if the equilibrium is relevant to multifragmentation reactions the achievement can be incomplete. Several aspects are expected to reflect the reaction dynamics, such as the pre-equilibrium emissions of light particles, the collective flow, and the expansion of the system [23, 24, 25, 26, 27].

The aim of this paper is to investigate whether the concept of equilibrium is relevant in multifragmentation, and if so, in what sense. We examine the achievement of equilibrium in multifragmentation reactions simulated by antisymmetrized molecular dynamics (AMD) [28, 29, 30]. AMD is a microscopic dynamical model based on the degrees of freedom of interacting nucleons. AMD is a suitable model for this study for the following reasons: It has been shown that various aspects of experimental data are reproduced by applying AMD to nuclear reactions [23, 28, 29, 30, 31, 32, 33, 34, 35, 36]. It has been also argued that the quantum and fermionic statistical properties of nuclear systems are correctly described by AMD if an appropriate quantum branching process is taken 
into account $[37,38,39,40]$. Furthermore, we can construct microcanonical equilibrium ensembles for given energies and volumes by solving the AMD equation of motion of a manynucleon system confined in a container for a long time [41]. By extracting temperature and pressure from these equilibrium ensembles and interpolating these data, we have drawn the constant-pressure caloric curves to show that negative heat capacity, which is a signal of the phase transition in finite systems [18, 42, 43], appears in the obtained result.

To investigate the relevance of equilibrium in multifragmentation, we employ the following steps. We perform the AMD simulation for very central ${ }^{40} \mathrm{Ca}+{ }^{40} \mathrm{Ca}$ collisions at 35 $\mathrm{MeV} /$ nucleon. The reaction ensemble at each reaction time $t$ is constructed by collecting the many-nucleon states at the time $t$ from different events. We compare this reaction ensemble with an equilibrium ensemble with appropriately chosen energy and volume. If we are able to find an equilibrium ensemble that is equivalent to the reaction ensemble, we may be able to discuss the connection between the multifragmentation data and statistical properties of nuclear matter. This subject has been studied by Raduta et al. [44, 45]. They have compared an ensemble obtained by the stochastic mean-field approach [46] which is a BUU-type transport model with that obtained by the microcanonical multifragmentation model [47] which is a statistical model. In contrast, we use the same version of AMD to describe both dynamical and equilibrium situations so that we can compare the reaction and equilibrium ensembles directly without being affected by the model difference.

This paper is organized as follows. In Sec. III the framework of AMD, which is used to simulate reaction and equilibrium systems, is explained. In Sec. III we show the results of the AMD simulation for the ${ }^{40} \mathrm{Ca}+{ }^{40} \mathrm{Ca}$ collisions at 35 $\mathrm{MeV} /$ nucleon, which have been already studied with another version of AMD [36]. One of the purposes of this section is to ensure that the modifications introduced in Ref. [41] for the application to statistical calculations do not spoil the good reproduction of the reaction data. Limiting the discussion to the very central reaction, we also argue the time evolution of the reaction system by showing the fragment observables. In Sec. IV we show results of the statistical calculation for an equilibrium system with 18 protons and 18 neutrons which is the same system as Ref. [41]. It is confirmed that negative heat capacity appears in the constant-pressure caloric curves although several modifications are introduced in this paper. In Sec. V we compare the ensembles obtained by the dynamical simulation (Sec. III) and obtained by the statistical calculations with various conditions of volume and energy (Sec.IV), and discuss whether the concept of equilibrium is relevant to the multifragmentation reaction. Section $\nabla I$ is devoted to a summary and future perspectives.

\section{FRAMEWORK OF AMD TIME EVOLUTION}

In this section, we present our AMD framework to calculate the time evolution of many-nucleon systems. We basically follow the framework of Ref. [41], although several modifica- tions are introduced in the present work. We simulate both a multifragmentation reaction (Sec. III) and an equilibrium system (Sec.IV with the same AMD model.

The wave function of an $A$-nucleon system $|\Psi(t)\rangle$ that evolves with time $t$ according to the many-body Hamiltonian is given by a superposition of various reaction channels. As it is impossible to follow the exact time evolution of $|\Psi(t)\rangle$ in practice, in the AMD formalism we approximate the manybody density matrix $|\Psi(t)\rangle\langle\Psi(t)|$ by an ensemble of AMD wave functions $|\Phi(Z)\rangle$ as

$$
|\Psi(t)\rangle\langle\Psi(t)| \approx \int \frac{|\Phi(Z)\rangle\langle\Phi(Z)|}{\langle\Phi(Z) \mid \Phi(Z)\rangle} w(Z, t) d Z,
$$

where $w(Z, t)$ is the weight factor for each reaction channel at time $t$. This approximation implies that we incorporate the existence of various reaction channels while we ignore the interference between channels since it is unimportant for practical purposes (decoherence).

AMD uses a single Slater determinant of Gaussian wave packets as a channel wave function

$$
\left\langle\mathbf{r}_{1} \cdots \mathbf{r}_{A} \mid \Phi(Z)\right\rangle=\operatorname{det}\left[\varphi_{\mathbf{Z}_{i}}\left(\mathbf{r}_{j}\right) \chi_{\alpha_{i}}(j)\right],
$$

where the spatial wave functions of nucleons $\varphi_{\mathbf{Z}}$ are given by

$$
\left\langle\mathbf{r} \mid \varphi_{\mathbf{Z}}\right\rangle=\left(\frac{2 v}{\pi}\right)^{3 / 4} \exp \left[-v\left(\mathbf{r}-\frac{\mathbf{Z}}{\sqrt{v}}\right)^{2}\right]
$$

and $\chi_{\alpha}$ denotes the spin-isospin wave function, $\chi_{\alpha}=p \uparrow$, $p \downarrow, n \uparrow$, and $n \downarrow$. The AMD wave function $|\Phi(Z)\rangle$ is the many-nucleon state parametrized by a set of complex variables $Z \equiv\left\{\mathbf{Z}_{i}\right\}_{i=1, \ldots, A}$. The real and the imaginary parts of $\mathbf{Z}$ correspond to the centroids of the position and the momentum of each wave packet, respectively, if the antisymmetrization effect is ignored. The width parameter $v$ is treated as a constant parameter common to all the wave packets and $v=0.16 \mathrm{fm}^{-2}$ is utilized in this paper, which has been adjusted to reasonably describe ground states of light nuclei such as ${ }^{16} \mathrm{O}$. It is shown that the binding energies of nuclei in a wide range of the nuclear chart are reproduced well with appropriate effective interactions [30,48]. This choice of channel wave function is suitable for the simulation of multifragmentation reactions, where each single-particle wave function should be localized within a fragment. Besides, the AMD wave function $|\Phi(Z)\rangle$ contains many quantum features owing to antisymmetrization and so is even utilized for nuclear structure studies [49].

According to Eq. (1), the time evolution of the $A$-nucleon system may be determined by calculating the time evolution of the weight factor for each channel $w(Z, t)$. Alternatively we take another viewpoint that the parameters $Z$ of the wave function $|\Phi(Z)\rangle$ are stochastic time-dependent variables $Z(t)$ and the time evolution of the many-nucleon state is given by the ensemble of the various trajectories. The stochastic time evolution of $Z(t)$ should be considered as the quantum branching from a channel $|\Phi(Z)\rangle$ to many other channels $\left|\Phi\left(Z_{1}^{\prime}\right)\right\rangle$, $\left|\Phi\left(Z_{2}^{\prime}\right)\right\rangle, \ldots$ 


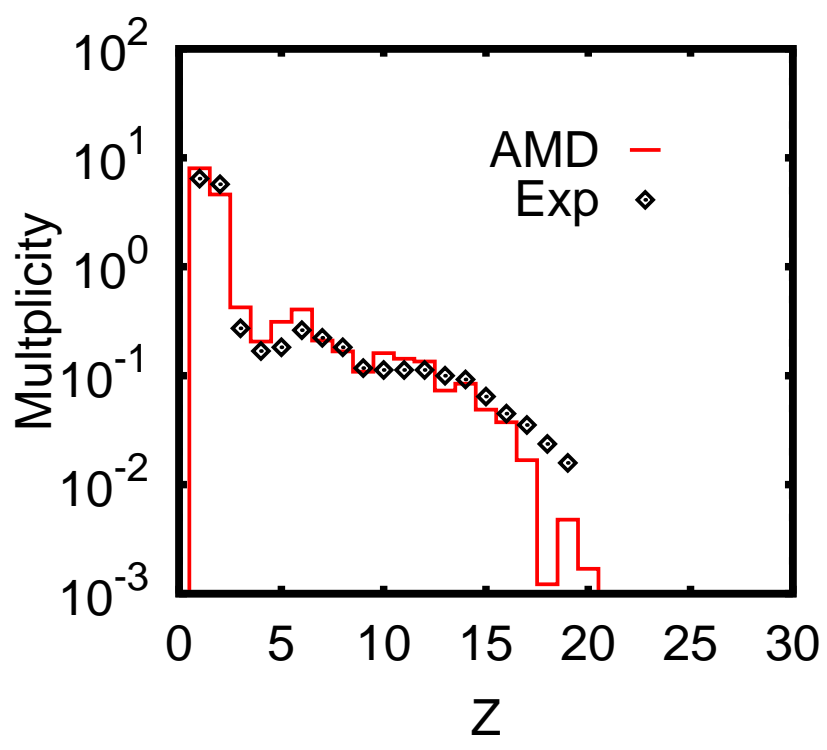

FIG. 2: (Color online) The fragment charge distribution of the reaction ${ }^{40} \mathrm{Ca}+{ }^{40} \mathrm{Ca}$ at $35 \mathrm{MeV} /$ nucleon simulated by AMD (full line) compared with the experimental data of Hagel et al. [51] (points).

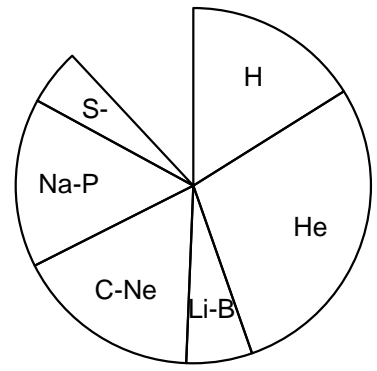

EXP

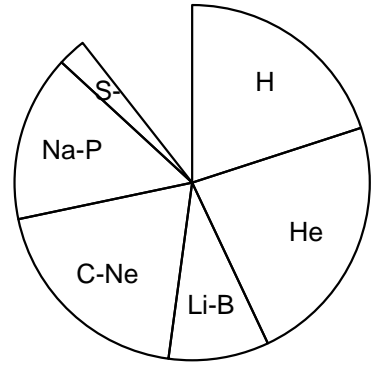

AMD
FIG. 3: Partition of total charge into fragments at the final state of the reaction ${ }^{40} \mathrm{Ca}+{ }^{40} \mathrm{Ca}$ at $35 \mathrm{MeV} /$ nucleon: the experimental data of Hagel et al. [51] (left) and the AMD result (right).

[51]. In Fig. 3] we also show how the total charge of the system is distributed in fragments in the final state. The experimental data show that $20 \%$ of protons are emitted as protons, deuterons, and tritons, $30 \%$ of protons are contained in $\mathrm{He}$ isotopes, and the rest of the protons are contained in heavier fragments. The features of the experimental data are reproduced by AMD well, as we see in Figs. 2 and 3 Reasonable reproduction of the fragments with $Z=1$ and 2 is obtained.

In this paper, we have chosen the coherence time parameter $\tau_{0}$ to be $5 \mathrm{fm} / c$ [Eq. (5)]. No significant difference is seen even if we take $\tau_{0}$ to be a half or twice this choice. When we take $\tau_{0}$ to be much longer such as $\tau_{0} \sim 100 \mathrm{fm} / c$, excessive production of heavy fragments is observed and consequently the amounts of the fragments around the B-Ne region are underestimated compared with the experimental data. The same trend has been seen when we use the AMD model described in Ref. [41]. This can be understood because the treatment

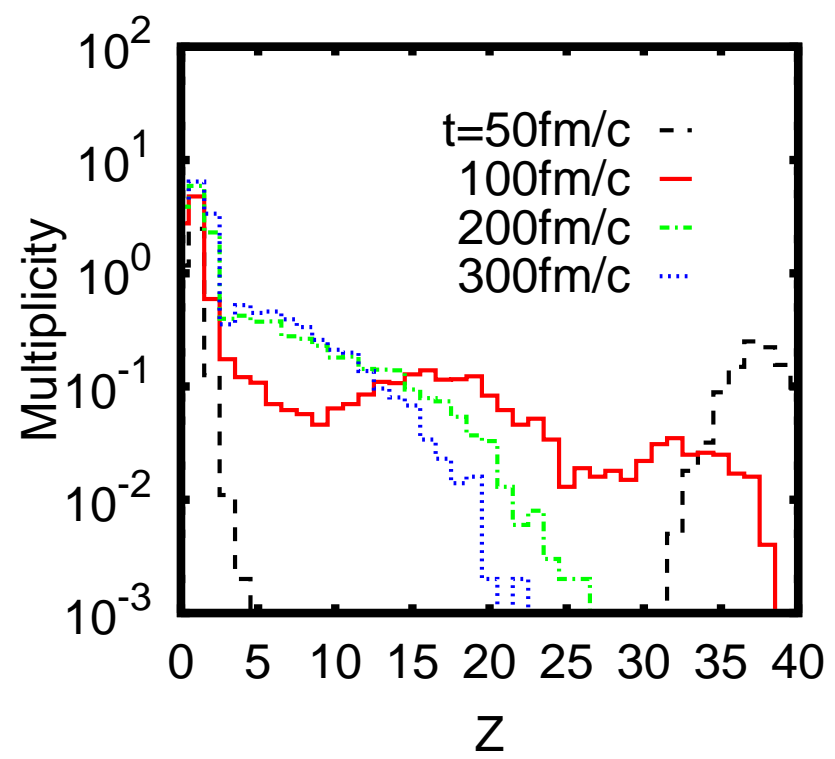

FIG. 4: (Color online) The fragment charge distribution of the very central $\left(b_{\text {imp }}=0 \mathrm{fm}\right)$ reaction ${ }^{40} \mathrm{Ca}+{ }^{40} \mathrm{Ca}$ at $35 \mathrm{MeV} /$ nucleon at four reaction times $t=50 \sim 300 \mathrm{fm} / c$.

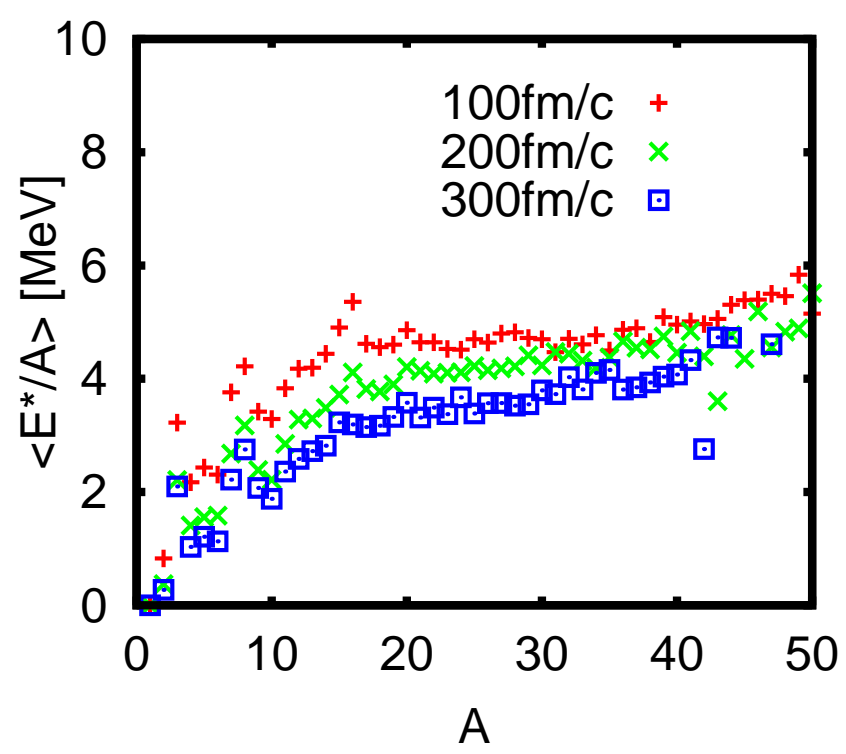

FIG. 5: (Color online) The average excitation energy of fragments as a function of the fragment mass number $A$ for the very central $\left(b_{\mathrm{imp}}=\right.$ $0 \mathrm{fm}$ ) reaction ${ }^{40} \mathrm{Ca}+{ }^{40} \mathrm{Ca}$ at $35 \mathrm{MeV} /$ nucleon at three reaction times $t=100 \sim 300 \mathrm{fm} / c$.

in Ref. [41] corresponds to a relatively weak decoherence (a long coherence time) as explained in the Appendix.

Let us concentrate our arguments on the time evolution of several observables for very central reaction events $\left(b_{\text {imp }}=0\right.$ $\mathrm{fm})$. The fragment charge distribution and the average excitation energy as a function of the fragment mass number are 
shown for several reaction times in Figs. 4 and 5 respectively, by identifying fragments with $r_{\text {frag }}=3 \mathrm{fm}$. The fragments identified in this way are not necessarily related to the fragments at the end of the reaction. Nevertheless, these quantities are helpful to understand the change of the reaction system along the time evolution. (The choice of the parameter $r_{\text {frag }}=3 \mathrm{fm}$ is taken to identify fragments even at early stages of the reaction, but $r_{\text {frag }}=3 \mathrm{fm}$ seems to be too small to identify the realistic fragments at time $t=300 \mathrm{fm} / c$.)

Isolated nucleons and light fragments are identified even at a very early stage of the reaction $(t=50 \mathrm{fm} / c)$; these are interpreted as pre-equilibrium emissions of light particles. The heavy fragments $Z>20$ are negligible at the truncation time $(t=300 \mathrm{fm} / c)$. The average excitation energies per nucleon of the fragments $A \geq 15$ are as high as about $5 \mathrm{MeV}$ at $t=100$ $\mathrm{fm} / c$ and decrease to about $4 \mathrm{MeV}$ at $t=300 \mathrm{fm} / c$.

In many very central reaction events, the produced fragments seem to be divided into two groups, projectile-like and target-like groups, at the late stage of the reaction (Fig. 1). Therefore, the two separate equilibrium systems of about half size will be more relevant to this reaction system rather than a single large equilibrium system, if the concept of equilibrium is relevant to this reaction in any sense.

\section{APPLICATION TO STATISTICAL CALCULATIONS}

We are able to study the statistical properties of manynucleon systems in equilibrium by using AMD as in Ref. [41]. We calculate the time evolution of the system of $A_{\text {total }}$ nucleons ( $N_{\text {total }}$ neutrons and $Z_{\text {total }}$ protons) confined in a spherical container of radius $r_{\text {wall }}$ for a long time. We regard the

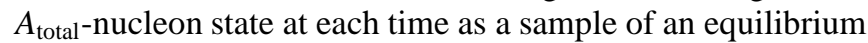
ensemble. The total energy $E_{\text {total }}$ of the system is conserved throughout the time evolution so that the obtained ensemble is a microcanonical ensemble specified by the total energy $E_{\text {total }}$, the volume $V_{\text {total }}=\frac{4}{3} \pi r_{\text {wall }}^{3}$ and the number of nucleons $A_{\text {total }}\left(Z_{\text {total }}, N_{\text {total }}\right)$. By extracting statistical information (temperature $T$ and pressure $P$ ) from the ensembles, we can construct caloric curves $T\left(E_{\text {total }}, P\right)$.

We utilize the same AMD model used to simulate the reaction ${ }^{40} \mathrm{Ca}+{ }^{40} \mathrm{Ca}$ at $35 \mathrm{MeV} /$ nucleon in the previous section to study the equilibrium system of $\left(Z_{\text {total }}, N_{\text {total }}\right)=(18,18)$, which is the same system as studied in Ref. [41]. We calculate ensembles for various energies $E_{\text {total }}^{*} / A_{\text {total }}=5 \sim 8 \mathrm{MeV}$ and volumes $r_{\text {wall }}=5 \sim 15 \mathrm{fm}\left(V_{\text {total }} / V_{0}=2.5 \sim 67\right)$. $E_{\text {total }}^{*}$ stands for the excitation energy relative to the ground state of the ${ }^{36}$ Ar nucleus $\left(E_{\text {g.s. }}=-8.9 A_{\text {total }} \mathrm{MeV}\right)$ and $V_{0}=A_{\text {total }} / \rho_{0}$ corresponds to the volume for the system with normal nuclear matter density $\rho_{0}$. The obtained constant-pressure caloric curves are shown in Fig. 6 Although several changes (explained in Sec. III and the Appendix) have been introduced in this paper, the characteristic feature of the phase transition in finite systems [18, 42, 43], namely negative heat capacity, can be recognized in the constant-pressure caloric curves with $P \leq 0.3 \mathrm{MeV}$, as has been seen in Ref. [41]. The caloric curve for the liquid phase, namely the line obtained by connecting the leftmost points of the constant-pressure caloric

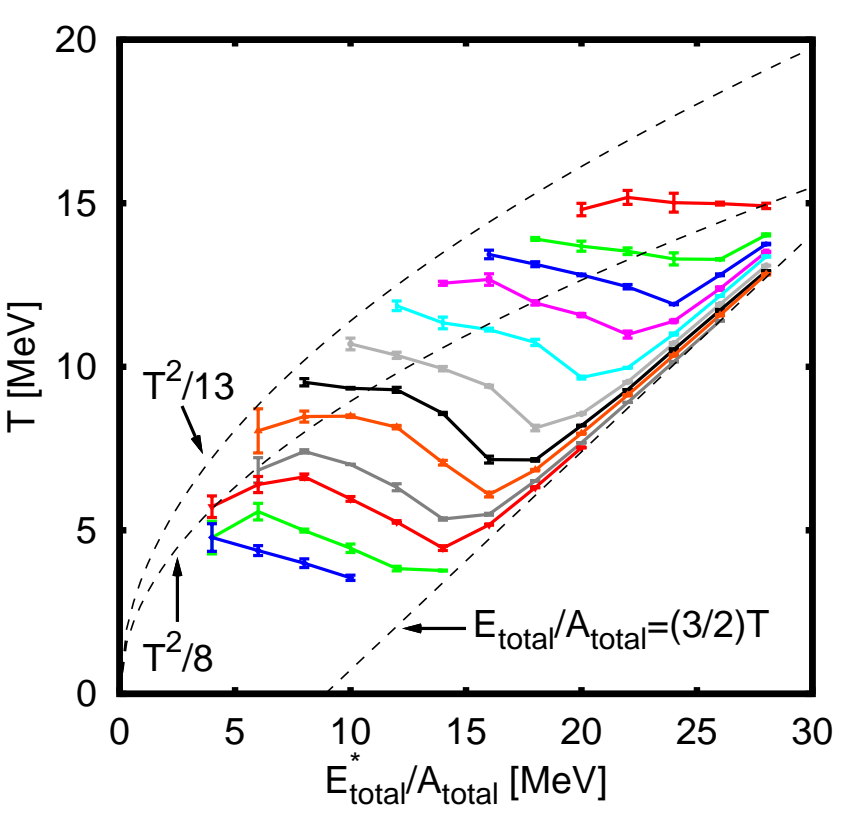

FIG. 6: (Color online) The constant-pressure caloric curves for the $A_{\text {total }}=36\left(N_{\text {total }}=18, Z_{\text {total }}=18\right)$ system obtained by AMD. The lines correspond to the pressure $P=0.005,0.01,0.02,0.03,0.05$, $0.07,0.10,0.15,0.20,0.25,0.30$ and $0.40 \mathrm{MeV} / \mathrm{fm}^{3}$ from the bottom upward. Statistical uncertainties are shown by error bars. The curves of $E_{\text {total }}^{*} / A_{\text {total }}=T^{2} /(8 \mathrm{MeV})$ and $E_{\text {total }}^{*} / A_{\text {total }}=T^{2} /(13 \mathrm{MeV})$, and the line of $E_{\text {total }} / A_{\text {total }}=\left(E_{\text {total }}^{*}+E_{\text {g.s. }}\right) / A_{\text {total }}=\frac{3}{2} T$ are drawn for comparison.

curves, shifted slightly left compared with the result of Ref. [41] and, connected to that, the critical point seems to be affected slightly. This is mostly due to the change of decoherence, as explained in Sec. VB in Ref. [41].

The created equilibrium ensembles in this section are utilized in Sec.V

\section{COMPARISON BETWEEN A DYNAMICAL SIMULATION AND STATISTICAL CALCULATIONS}

In this section, we compare two ensembles - a reaction ensemble and an equilibrium ensemble - to study whether the concept of equilibrium is relevant in multifragmentation reactions.

A reaction ensemble An ensemble obtained by collecting the states at a certain reaction time from many events of a dynamical multifragmentation reaction simulated by AMD (Sec. III). The reaction ensemble is specified by the reaction time $t$, and we consider the ensembles obtained from the reaction ${ }^{40} \mathrm{Ca}+{ }^{40} \mathrm{Ca}$ at $35 \mathrm{MeV} /$ nucleon in Sec. III We use only very central reaction events $\left(b_{\text {imp }}=0 \mathrm{fm}\right)$.

An equilibrium ensemble: An ensemble obtained by calculating the time evolution of a many-nucleon system in a 
container for a long time by AMD and regarding a state at each time as a sample (Sec. IV). The equilibrium ensemble here is a microcanonical ensemble specified by the total energy $E_{\text {total }}$, container volume $V_{\text {total }}$, and number of nucleons $A_{\text {total }}\left(Z_{\text {total }}, N_{\text {total }}\right)$. We consider the system of $\left(Z_{\text {total }}, N_{\text {total }}\right)=(18,18)$ studied in Sec. IV

We utilize the same AMD model to calculate both situations so that we are able to compare the reaction and equilibrium ensembles without ambiguities.

The comparison of the reaction and equilibrium ensembles is performed by calculating the same observables for both ensembles. In this paper, the fragment charge distribution $Y_{Z}$ and the average excitation energy as a function of the fragment mass number $\left\langle E^{*} / A\right\rangle_{A}$ are chosen as the observables ("fragment observables"). To make a detailed comparison with many observables, we introduce three classes of fragment observables by choosing different values of the fragment identification parameter $r_{\text {frag }}: r_{\text {frag(1) }}=3 \mathrm{fm}, r_{\text {frag(2) }}=2.5 \mathrm{fm}$ and $r_{\text {frag(3) }}=2 \mathrm{fm}$ (see Sec. III). The fragment observables with different $r_{\text {frag }}$ can be regarded as different observables for the comparison of ensembles.

For a given reaction time $t$, we compute a quantity

$$
\begin{aligned}
\delta^{2} & =\frac{1}{3 \times 13}\left[\sum_{i=1}^{3} \sum_{Z=4}^{16}\left\{\ln Y_{Z \text { react }}^{(i)}-\ln \left(\mathcal{N} Y_{Z \text { equil }}^{(i)}\right)\right\}^{2}\right] \\
& +\frac{1}{3 \times 14}\left[\frac{1}{\epsilon^{2}} \sum_{i=1}^{3} \sum_{A=2}^{15}\left\{\left\langle E^{*} / A\right\rangle_{A \text { react }}^{(i)}-\left\langle E^{*} / A\right\rangle_{A \text { equil }}^{(i)}\right\}^{2}\right]
\end{aligned}
$$

and search the equilibrium ensemble that gives the minimum value of $\delta^{2}$. Here $Y_{Z}$ and $\left\langle E^{*} \mid A\right\rangle_{A}$ are the yield of the fragments with the charge number $Z$ and the average excitation energy of the fragments with mass number $A$, respectively. The subscripts "react" and "equil" indicate that the observables for the reaction ensemble and for the equilibrium ensemble, respectively. The superscript ( $i$ ) denotes that the observables are calculated with the fragments identified by $r_{\text {frag }(i)}$. The factor $\epsilon$ is a dimensional constant of energy and taken as $1 \mathrm{MeV}$ in this paper. The factor $\mathcal{N}$ is a normalization constant that is optimized to give the minimum value of $\delta^{2}$. The yields $Y_{Z}$ of the fragments with $Z=1 \sim 3$ are omitted to compute $\delta^{2}$ to avoid the effect of pre-equilibrium emissions.

In Sec. III we have seen that the reaction system seems to be composed of two separate equilibrium systems if equilibrium is relevant to this reaction. The system $\left(Z_{\text {total }}, N_{\text {total }}\right)=$ $(18,18)$ we studied in Sec. IV is about half the size of the reaction system. We therefore compare the reaction ensemble of Sec. III and the equilibrium ensembles of Sec. IV A value of $\mathcal{N} \approx 2$ is expected to compare the equilibrium system of $\left(Z_{\text {total }}, N_{\text {total }}\right)=(18,18)$ with the reaction system of ${ }^{40} \mathrm{Ca}+{ }^{40} \mathrm{Ca}$. In early stages of the reaction, a heavy fragment with $Z>20$ is identified when the projectile-like and target-like groups overlap spatially. It is not appropriate to compare such situations with an equilibrium ensemble with $\left(Z_{\text {total }}, N_{\text {total }}\right)=(18,18)$. We therefore exclude from the reaction ensemble the states in which a heavy fragment with $Z>20$ is identified with $r_{\text {frag(1) }}=3 \mathrm{fm}$. We start the com-
TABLE I: The state variables of the equilibrium ensemble that reproduces the fragment observables of the reaction ${ }^{40} \mathrm{Ca}+{ }^{40} \mathrm{Ca}$ at 35 $\mathrm{MeV} /$ nucleon at each reaction time $t=80 \sim 300 \mathrm{fm} / c$.

\begin{tabular}{ccccccc}
\hline \hline $\begin{array}{c}t \\
{[\mathrm{fm} / c]}\end{array}$ & $\begin{array}{c}E_{\text {total }}^{*} / A_{\text {total }} \\
(\mathrm{MeV})\end{array}$ & $V_{\text {total }} / V_{0}$ & $\begin{array}{c}T \\
(\mathrm{MeV})\end{array}$ & $\begin{array}{c}P \\
\left(\mathrm{MeV} / \mathrm{fm}^{3}\right)\end{array}$ & $\mathcal{N}$ & $\delta^{2}$ \\
\hline 80 & 6.9 & 3.3 & 8.1 & 0.042 & 1.4 & 0.46 \\
100 & 6.5 & 3.9 & 7.1 & 0.029 & 2.0 & 0.18 \\
120 & 6.3 & 5.0 & 6.1 & 0.019 & 2.1 & 0.12 \\
140 & 6.1 & 6.2 & 5.9 & 0.013 & 2.0 & 0.13 \\
160 & 5.9 & 6.4 & 5.6 & 0.011 & 2.0 & 0.12 \\
180 & 5.7 & 6.6 & 5.4 & 0.010 & 2.0 & 0.13 \\
240 & 5.4 & 9.2 & 4.7 & 0.007 & 1.8 & 0.11 \\
300 & 5.3 & 13.2 & 4.1 & 0.005 & 1.9 & 0.15 \\
\hline \hline
\end{tabular}

parison after the reaction time $t=80 \mathrm{fm} / c$ at which we find a significant number of adopted states.

The reaction ensembles at the time $t=80,100,120,140$, $160,180,240$ and $300 \mathrm{fm} / c$ are compared with the equilibrium ensembles $E_{\text {total }}^{*} / A_{\text {total }}=5 \sim 8 \mathrm{MeV}$ and $r_{\text {wall }}=5 \sim 9$ fm $\left(V_{\text {total }} / V_{0}=2.5 \sim 14\right)$. When the energy of the equilibrium system $E_{\text {total }}^{*} / A_{\text {total }}$ is varied, a large change is observed in $\left\langle E^{*} / A\right\rangle_{A}$. However, when the volume of the equilibrium system $V_{\text {total }}$ is varied, the change of the shape of $Y_{Z}$ is noticed. Therefore, by reproducing $Y_{Z}$ and $\left\langle E^{*} / A\right\rangle_{A}$, we are able to find an equilibrium ensemble (specified by $E_{\text {total }}$ and $V_{\text {total }}$ ) that reproduces the reaction ensemble, if it exists. The observables for the equilibrium ensemble depend on the size of the system, but we have confirmed that this dependence is compensated by the freedom of the normalization factor $\mathcal{N}$ when we change the number of nucleons in the equilibrium system to $A_{\text {total }}=20$.

Figure 7 is the comparison of the observables between the reaction and equilibrium ensembles. The equilibrium ensemble is chosen to minimize the $\delta^{2}$ value for the reaction ensemble at each reaction time $t=80 \sim 300 \mathrm{fm} / c$. Overall features of the fragment observables of both ensembles agree well at every reaction time except for small details. The comparisons of $\left\langle E^{*} / A\right\rangle_{A}$ for the fragments identified with $r_{\text {frag }}=2.5$ and 2 $\mathrm{fm}$ are not shown in Fig. 7] but the agreement between the two ensembles are as good as in the case of $r_{\text {frag }}=3 \mathrm{fm}$. Table $\llbracket$ shows the energy, volume, temperature and pressure of the best-fit equilibrium ensembles. The normalization factor $\mathcal{N}$ and $\delta^{2}$ values are also listed in the table. The larger volume is required to reproduce the later stage of the reaction while the energy per nucleon decreases gradually. As a consequence, the temperature and pressure of the system decrease along the reaction time. In the caloric curves of Fig. 8 we show the reaction path by connecting the points $\left(E_{\text {total }}^{*} / A_{\text {total }}, T\right)$ of the equilibrium systems corresponding to different reaction times. All these points seem to be located in the region of liquid-gas coexistence that includes the region of negative heat capacity, and therefore it seems that the fragmentation of this reaction is connected to the nuclear liquid-gas phase transition. 


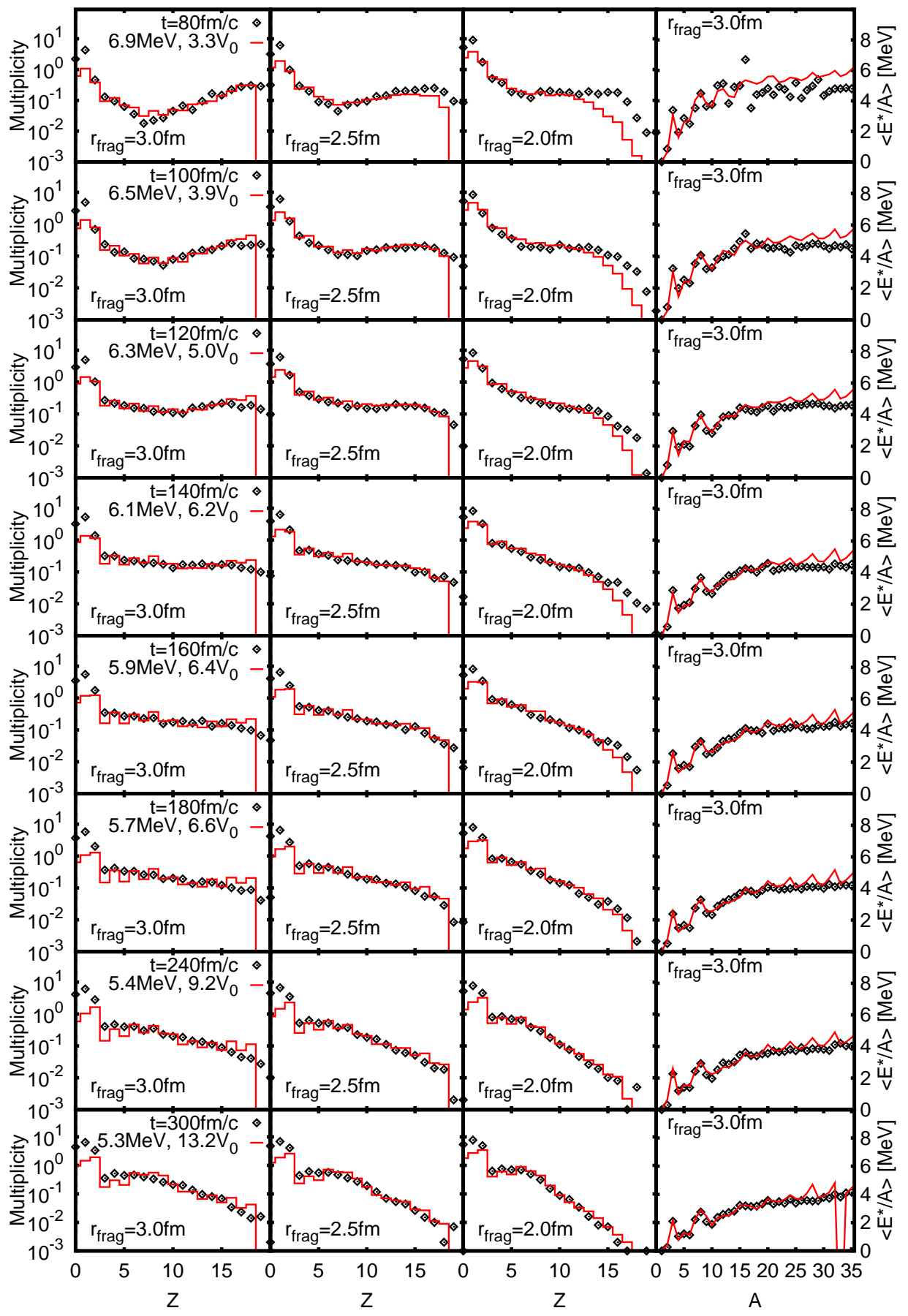

FIG. 7: (Color online) Comparison of the fragment charge distribution (left three columns) and the average excitation energy of fragments (rightmost column) of the reaction ensemble at each reaction time $t=80 \sim 300 \mathrm{fm} / c$ obtained from the very central $\left(b_{\text {imp }}=0 \mathrm{fm}\right)$ reaction ${ }^{40} \mathrm{Ca}+{ }^{40} \mathrm{Ca}$ at $35 \mathrm{MeV} /$ nucleon and those of the best-fit equilibrium ensemble of the system $\left(Z_{\text {total }}, N_{\text {total }}\right)=(18,18)$. The reaction time of the reaction ensemble and the energy and the volume of the equilibrium ensemble are shown in the leftmost column. The values of $r_{\text {frag }}$ for the fragment identification are indicated. 


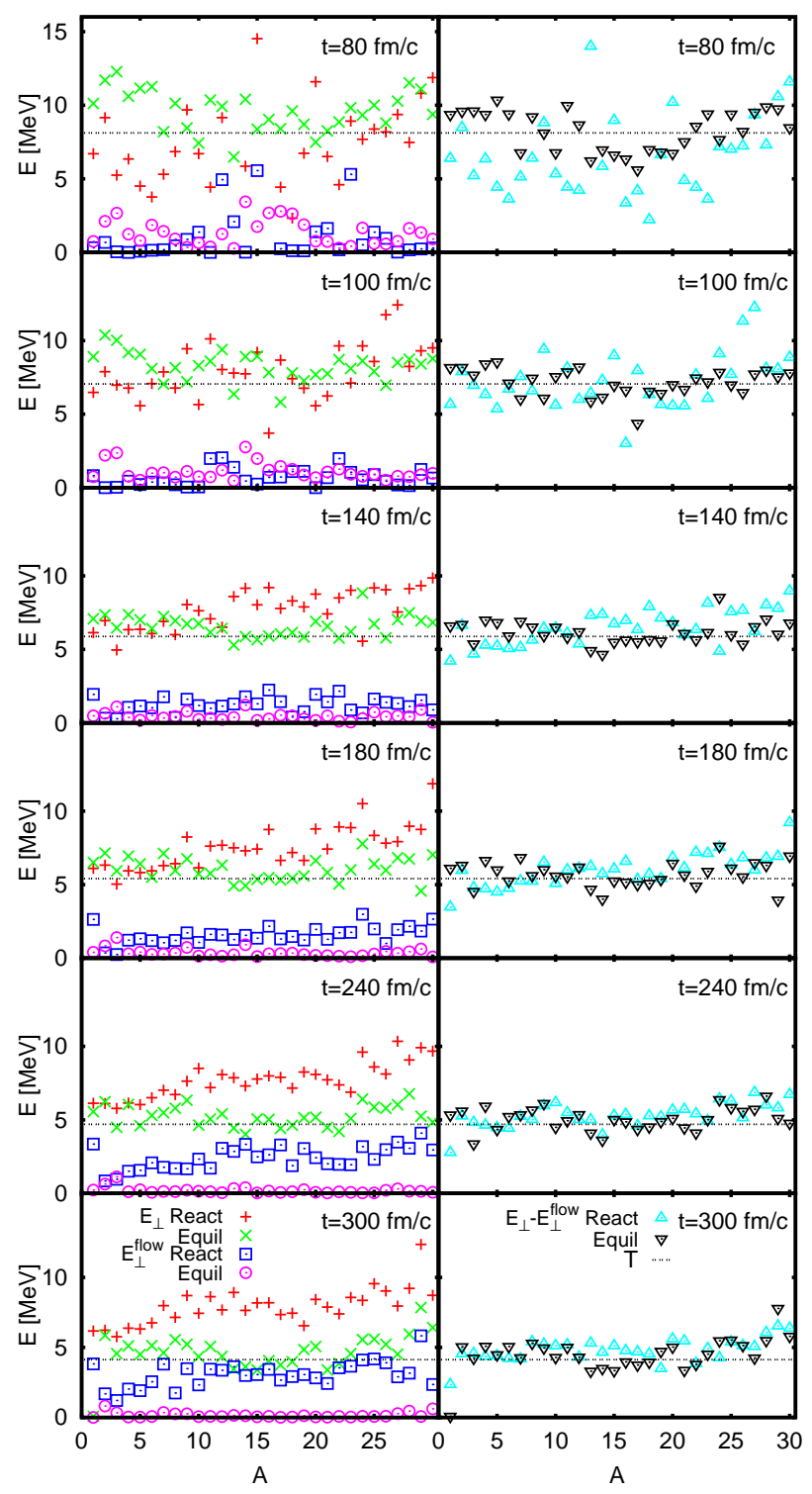

FIG. 9: (Color online) Comparison of the observables related to the fragment transverse momenta $\left(E_{\perp}\right.$ and $\left.E_{\perp}^{\text {flow }}\right)$ of the reaction ensemble ("React") at each reaction time $t=80 \sim 300 \mathrm{fm} / c$ with those of the corresponding equilibrium ensemble ("Equil"). $E_{\perp}$ and $E_{\perp}^{\text {flow }}$ are shown in the left column as functions of the fragment mass number $A$, and the difference $E_{\perp}-E_{\perp}^{\text {flow }}$ is shown in the right column. The dashed horizontal lines indicate the temperature $T$ of the equilibrium ensembles.

The comparison between the reaction and equilibrium ensembles is shown in the left panels of Fig. 9 for the observables $E_{\perp}(A)$ and $E_{\perp}^{\text {flow }}(A)$ at various reaction times. Large differences between the ensembles are found for these observables especially at the late stage of the reaction. For instance, nonnegligible $E_{\perp}^{\text {flow }}$ for the reaction ensemble (shown by squares) is noticed at $t \gtrsim 140 \mathrm{fm} / c$, whereas $E_{\perp}^{\text {flow }}$ for the equilibrium (shown by circles) is almost zero for all the times as it should be for equilibrated systems. (At $t=80 \mathrm{fm} / c$, the statistical results are insufficient to draw conclusions.) However, the difference $E_{\perp}(A)-E_{\perp}^{\text {flow }}(A)$ agrees quite well between the reaction and equilibrium ensembles, as shown in the right panels of Fig. 9 at all the shown times. Furthermore, $E_{\perp}(A)-E_{\perp}^{\text {flow }}(A)$ has nearly no dependence on the mass number $A$, and its value almost agrees with the value of the temperature $T$ of the equilibrium ensemble shown by the horizontal line at each reaction time. This surprising agreement also suggests a consistency of the model, since the temperature has been extracted from an equilibrium ensemble without using the information of fragment kinetic energies. Thus the reaction results for the observables related to the fragment momenta seem to be still consistent with the equilibrium results if we subtract the flow effects from the reaction results.

Second, we estimate the size of the reaction system. The volume listed in Table 1 is that of the equilibrium system that gives the best fit for the fragment observables of the reaction system. However, the real volume of the reaction system may be different from this. To estimate the size of the reaction system, we compute the root mean square radius of the total system in the $x^{\prime} y^{\prime}$-plane

$$
R_{\perp}^{\text {system }}=\left\langle\sqrt{\frac{1}{N_{\mathcal{S}(A>5)}} \sum_{i \in \mathcal{S}(A>5)}\left(R_{i x^{\prime}}^{2}+R_{i y^{\prime}}^{2}\right)}\right\rangle
$$

by using the nucleon positions $R_{i \sigma}\left(\sigma=x^{\prime}, y^{\prime}, z^{\prime}\right)$, where $\mathcal{S}(A>5)$ denotes the nucleons that belong to the fragments with mass number greater than 5 , and $N_{\mathcal{S}(A>5)}$ is the number of these nucleons in each event. The nucleons that belong to light fragments $(A \leq 5)$ are omitted from the calculation in Eq. (11) to minimize the effect of pre-equilibrium emissions. The results are given in Fig. 10 The radius $R_{\perp}^{\text {system }}$ of the reaction ensemble is larger than that of the equilibrium ensemble at all the reaction times and the difference increases with time. For the reaction ensemble, the system may be more extended along the beam axis owing to the memory of reaction dynamics and then the difference of the volume between the reaction and equilibrium systems will be more prominent. Therefore, the difference of $R_{\perp}^{\text {system }}$ shown in Fig. 10] suggests that the real volume of the reaction system is larger than the volume of the corresponding equilibrium system typically by $50 \%$ or more. Conversely, if the real volume is required to agree between reaction and equilibrium ensembles, any good fitting of $Y_{Z}$ will not be obtained, because of the strong volume dependence of $Y_{Z}$ of the equilibrium system as can be seen from Fig. 7 (The dependence of $Y_{Z}$ on the system energy is weak, as mentioned eqrlier.) Thus the usual static equilibrium at each instant is not realized. This may be because fragments are formed in a dynamically expanding system and the observables of fragments recognized at a reaction time $t$ may be reflecting the history of the state of the system before $t$ rather than the volume at that instant $t$.

Third, we calculate the root mean square radii of fragments $R_{\text {rms }}(A)$ for the reaction and equilibrium ensembles to investigate whether the properties of the created fragments in the reaction system are the same as those in the equilibrium system (Fig. 11]. We find that $R_{\mathrm{rms}}(A)$ of intermediate-mass fragments $(A=6 \sim 20)$ for the reaction ensemble are system- 


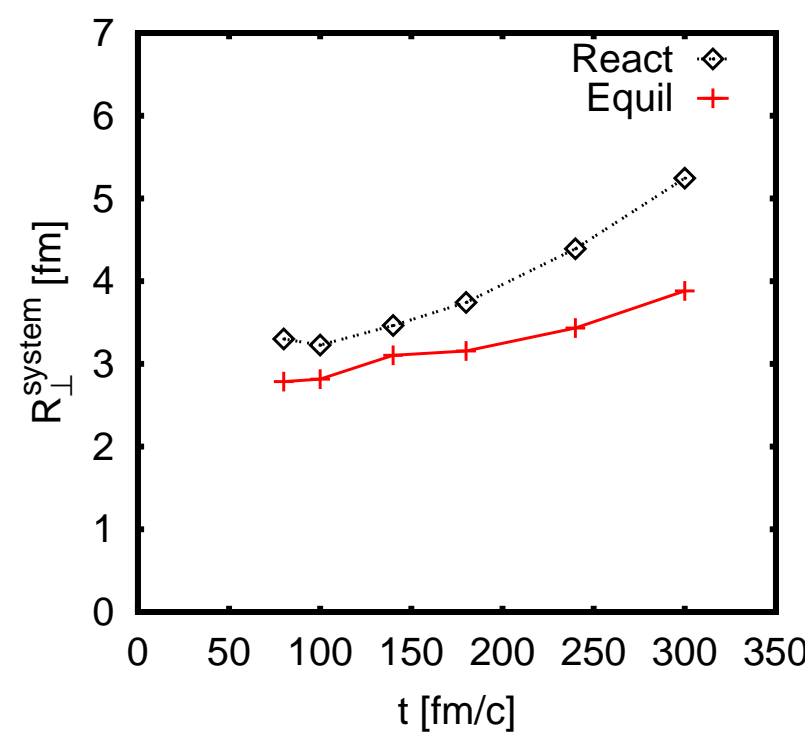

FIG. 10: (Color online) The root mean square radius $R_{\perp}^{\text {system }}$ of the total system in the $x^{\prime} y^{\prime}$-plane for the reaction ensemble ("React") and the corresponding equilibrium ensemble ("Equil") as functions of the reaction time.

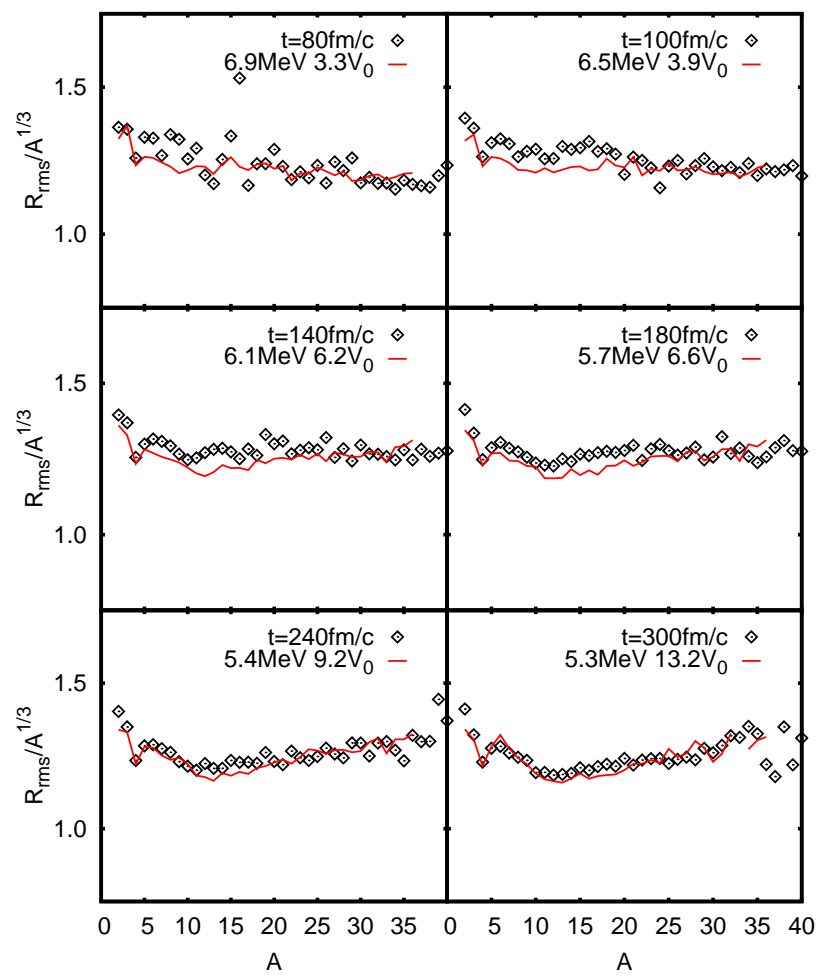

FIG. 11: (Color online) The fragment root mean square radius $R_{\mathrm{rms}}(A)$ divided by $A^{1 / 3}$ plotted as a function of the fragment mass number $A$ for the reaction ensemble at each reaction time $t=80 \sim$ $300 \mathrm{fm} / c$ and for the corresponding equilibrium ensemble.

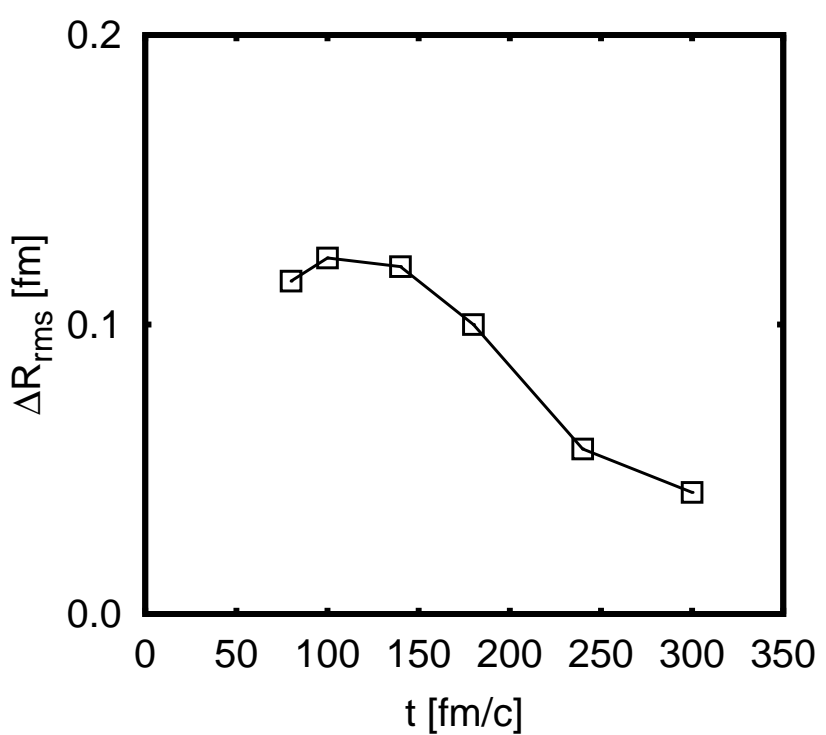

FIG. 12: The time dependence of the difference of the fragment root mean square radius between the reaction and corresponding equilibrium ensembles, averaged over the intermediate-mass fragments $(A=6 \sim 20)$.

atically (about $5 \%$ ) larger than those for the equilibrium ensemble. We also calculate the average of the difference of $R_{\mathrm{rms}}(A)$ between the ensembles over a range of intermediate mass fragments $(A=6 \sim 20)$;

$$
\Delta R_{\mathrm{rms}}=\frac{1}{15} \sum_{A=6}^{20}\left\{R_{\mathrm{rms}}^{\mathrm{react}}(A)-R_{\mathrm{rms}}^{\text {equil }}(A)\right\} .
$$

We plot $\Delta R_{\mathrm{rms}}$ as a function of the reaction time in Fig. 12 The difference is large at the early stage of the reaction $(t \sim 100 \mathrm{fm} / c)$ and reduces with time at the late stage of the reaction. In fact, the radii of the intermediate-mass fragments in the reaction ensemble at $t \sim 100 \mathrm{fm} / c$ are larger than those in any of the equilibrium ensembles that we have investigated. This may be an indication of the fragment formation mechanism in which the fragments in the reaction are made from the expanding dilute system where surface effects are less important. It may also be related to the finding that the symmetry energy extracted from the multifragmentation reactions shows almost no surface effect [53].

\section{SUMMARY}

In this paper, we have investigated the relevance of the equilibrium concept in multifragmentation by comparing reaction and equilibrium ensembles. The reaction ensemble at each reaction time $t$ is constructed by gathering the manynucleon states at time $t$ in AMD simulations of very central ${ }^{40} \mathrm{Ca}+{ }^{40} \mathrm{Ca}$ collisions at $35 \mathrm{MeV} /$ nucleon. The equilibrium ensemble is prepared by solving the AMD equation of motion of a many-nucleon system $\left(Z_{\text {total }}, N_{\text {total }}\right)=(18,18)$ confined 
in a container for a long time. We then compare the reaction ensemble at each $t$ with equilibrium ensembles at various conditions of volume and energy. We have used exactly the same AMD model in simulating both situations. To our knowledge, this is the first work that directly compares the multifragmentation reaction and the corresponding equilibrium system by describing both situations with one model.

The AMD model used in this paper has been modified from that in Ref. [41] to better incorporate the effect of decoherence. We have confirmed the validity of the current version of AMD by comparing the result of ${ }^{40} \mathrm{Ca}+{ }^{40} \mathrm{Ca}$ reactions at $35 \mathrm{MeV} /$ nucleon with the experimental data [51]. We have also confirmed that the constant-pressure caloric curves of the equilibrium system $\left(Z_{\text {total }}, N_{\text {total }}\right)=(18,18)$ constructed with the same AMD show negative heat capacity which is the signal of the phase transition in finite systems.

The comparison between the reaction and equilibrium ensembles has been performed by computing the fragment charge distribution and the average excitation energies of fragments (fragment observables) for both ensembles. We are able to find an equilibrium ensemble that reproduces overall features at each reaction time $t=80 \sim 300 \mathrm{fm} / c$. For the later stage of the reaction, an equilibrium ensemble with a larger volume and a slightly lower energy is required. This is consistent with the scenario that the system created by heavy-ion collisions cools during expansion. Unfortunately, it is difficult to identify the beginning and the end of the equivalence between the reaction and equilibrium systems, and it will be interesting to further develop the study to explore these. Experimentally, isotope thermometers have been utilized to extract the temperature from reactions [54, 55]. By comparing it with the temperature obtained by numerical simulation, it may be possible to identify the reaction stage relevant to the experimentally obtained isotope temperature.

The reaction ensembles have been constructed without any assumption of thermal equilibrium. Nevertheless, we can find an equilibrium ensemble that is almost equivalent to the reaction ensemble as far as the fragment observables are concerned at each reaction time after $t=80 \mathrm{fm} / c$. This is a rather surprising result, since there are certainly some observables that reflect the reaction dynamics. In fact, we have given several examples of the observables that show some discrepancy between the reaction and corresponding equilibrium ensembles. The fragment transverse kinetic energies are different from those of the equilibrium system, especially for the late stages of the reaction. However, the difference can be explained by simple flow effects. If the flow effects are subtracted, the fragment kinetic energies of the reaction system is still consistent with those of the equilibrium system. The size of the reaction system is larger than that of the equilibrium system. Namely, the real volume of the reaction system is larger than the volume assigned by fitting the fragment observables. The difference becomes larger at the later stages of the reaction. The usual static equilibrium at each instant is not realized since any equilibrium ensemble with the same volume as that of the reaction system cannot reproduce the fragment observables. The fragment radii in the reaction system are larger than those in the equilibrium system. The difference is large at the early stage of the reaction $(\sim 100 \mathrm{fm} / c)$ and decreases with time. This may be an indication of a fragment formation mechanism in which the fragments are made from an expanding dilute system in the reaction.

Only a small difference between the reaction and equilibrium ensembles is seen in the fragment observables studied in this paper. However, dynamical effects may become essential even for the fragment observables when the incident energy is increased or the impact parameter is varied. It has been suggested that neck formation play an important role in semiperipheral collisions [56], but in this paper we ignored this effect. It is an interesting question whether the equivalence between the multifragmentation reaction and the equilibrium system still holds under such circumstances. It is also interesting to compare observables such as the momentum distribution of fragments and the system size of multifragmentation reactions with those of the corresponding equilibrium systems in the explicit presence of expansion and flow effects [57, 58].

In this paper, we studied only one particular reaction, namely very central ${ }^{40} \mathrm{Ca}+{ }^{40} \mathrm{Ca}$ collisions at $35 \mathrm{MeV} /$ nucleon. The reaction mechanism changes from one reaction to another. It is therefore interesting to apply the same approach to other reactions, such as a reaction of heavier nuclei where creation of a single thermal source is expected [44, 45], and a reaction of nuclei with different isospin compositions where the occurrence of isospin diffusion has been claimed [59, 60]. It is important to explore the effects of various reaction parameters such as the reaction system, the incident energy and the impact parameter on the achievement of equilibrium. If the concept of equilibrium is relevant, it is interesting to explore how these parameters influence the parameters to specify the equilibrium system. This study will offer guidelines for combining experimental data of various heavy-ion collisions to construct, for example, equation of states and constant-pressure caloric curves.

\section{Acknowledgments}

The major part of this work has been done at Tohoku University as the Ph. D. study of T.F. T.F. acknowledges partial support from the ANR project NExEN (ANR-07-BLAN0256-02). This work is partly supported by the High Energy Accelerator Research Organization (KEK) as a supercomputer project.

\section{APPENDIX A: IMPROVED IMPLEMENTATION OF DECOHERENCE}

The reaction ${ }^{40} \mathrm{Ca}+{ }^{40} \mathrm{Ca}$ at $35 \mathrm{MeV} /$ nucleon has already been studied by AMD [23, 36] and it has been shown that several aspects of the experimental data [51] are nicely reproduced. The AMD model used in these studies adopts the instantaneous decoherence of the single-particle motion [36]. In contrast, in Ref. [41] and in this paper, we utilize the AMD model in which the coherence of the single-particle motions are kept for a finite duration. When we directly applied the 
AMD formalism given in Ref. [41] to the reaction ${ }^{40} \mathrm{Ca}+{ }^{40} \mathrm{Ca}$ at $35 \mathrm{MeV} /$ nucleon, excessive productions of heavy fragments are obtained and, connected to that, amounts of lighter fragments around the B-Ne region are underestimated compared with the experimental data. This is because the coherence time chosen by the formalism in Ref. [41] is too long and the effect of decoherence is hindered for some cases, and thus it fails to give enough quantum fluctuations to break the heavy fragments. A modification is necessary to better incorporate the effect of decoherence and reproduce the experimental data. This is rather technical but the summary is given in this Appendix.

In the AMD formalism, special care is taken for the nucleons that are almost isolated. For instance, the zero-point kinetic energies of these nucleons are subtracted since the wave functions of such nucleons should have sharp momentum distributions rather than Gaussian ones corresponding to the wave packet in Eq. (3). This change of interpretation is necessary for the consistency of Q-values of nucleon emissions and fragmentation [28, 29, 30] and is very important for the definition of temperature [41]. In Ref. [41], we judge the "degree of isolation" of the nucleon $k$ by introducing

$$
\mathcal{I}_{k}=\left[1-w\left(q_{k}\right)\right] \mathcal{I}_{k}^{(0)}+w\left(q_{k}\right)
$$

where $q_{k}$ counts the neighboring nucleons of the nucleon $k$ including itself, $w(q)$ is a continuous function from one when the number of neighboring nucleons $q_{k}$ is small $(q \lesssim 2.5)$ to zero, and $\mathcal{I}_{k}^{(0)}$ corresponds to the inverse number of the neighboring nucleons. Detailed definitions of these functions are given in Appendix A in Ref. [41].

In the AMD formalism, the phase-space distribution $g(x ; X, S)$ is considered to compute the time evolution of the mean-field propagation. The distribution for each nucleon $k$ is parametrized by

$$
\begin{aligned}
g\left(x ; X_{k}, S_{k}\right) & =\frac{1}{8 \sqrt{\operatorname{det} S}} \\
& \times \exp \left[-\frac{1}{2} \sum_{a, b=1}^{6} S_{k a b}^{-1}\left(x_{a}-X_{a}\right)\left(x_{b}-X_{b}\right)\right],
\end{aligned}
$$

where $x$ gives the six-dimensional phase-space coordinates

$$
x=\left\{x_{a}\right\}_{a=1, \ldots, 6}=\left\{\sqrt{v} \mathbf{r}, \frac{\mathbf{p}}{2 \hbar \sqrt{v}}\right\},
$$

and $S_{k}$ and $X_{k}$ specify the shape and the centroids of the distribution, respectively. $X_{k}$ is identified with the physical coordinate $\mathbf{W}_{k}$ [28, 29, 30]:

$$
X_{k}=\left\{X_{k a}\right\}_{a=1, \ldots, 6}=\left\{\operatorname{Re} \mathbf{W}_{k}, \operatorname{Im} \mathbf{W}_{k}\right\} .
$$

In Ref. [41], one condition was imposed on $g\left(x ; X_{k}, S_{k}\right)$ for each nucleon $k$ by using the degree of isolation $\mathcal{I}_{k}$. The condition was

$$
\operatorname{Tr}_{p} S_{k} \leq \frac{3}{4}\left(1-\mathcal{I}_{k}\right)
$$

where $\operatorname{Tr}_{p} S_{k}=S_{k 44}+S_{k 55}+S_{k 66}$ denotes the momentum spreading of the distribution $g\left(x ; X_{k}, S_{k}\right)$. Namely, if the lefthand side of Eq. A5 is getting larger than the right-hand side, $S_{k}$ was reduced to satisfy the equality of Eq. A5 and the reduced part was converted into a stochastic Gaussian fluctuation to the centroid $X_{k}$ (the details are explained in Sec. III in Ref. [41]). The purpose of this condition is to ensure full consistency of the energy conservation and to allow precise evaluation of the temperature. When a recovery of the phasespace distribution $g(x ; X, S)$ for nucleon $k$ took place as a result of decoherence, we replaced the shape of the distribution $S_{k}$ with

$$
S_{k a b}= \begin{cases}\frac{1}{4} & (a=b=1,2,3) \\ \frac{1}{4}\left(1-\mathcal{I}_{k}\right) & (a=b=4,5,6), \\ 0 & (a \neq b)\end{cases}
$$

where the momentum widths were chosen to be $\frac{1}{4}\left(1-\mathcal{I}_{k}\right)$ rather than the standard Gaussian width $1 / 4$ to satisfy Eq. A5.

The condition is arbitrary as long as $\operatorname{Tr}_{p} S_{k} \simeq \frac{3}{4}\left(1-\mathcal{I}_{k}\right)$ is satisfied for the nucleon $k$ that is utilized to measure the temperature. Unfortunately, it turns out that the condition A5 utilized in Ref. [41] tends to hinder the effect of decoherence unphysically at the surface of fragments. This is because $I_{k}$ increases close to unity when the nucleon $k$ is located near the surface of the fragment to which the nucleon $k$ belongs. The increase of $\mathcal{I}_{k}$ results in keeping $\operatorname{Tr}_{p} S_{k}$ small, even though the recovery of the phase-space distribution defined by Eq. A6 frequently occurs. There is no physical reason why the effect of decoherence is suppressed at the surface of fragments and it is more natural that the effect of decoherence for the nucleon $k$ is as large as those for the other nucleons belonging to the same fragment even though the nucleon $k$ is located near the surface. We thus introduce a new function

$$
\mathcal{I}_{k}^{*}=w\left(q_{k}\right)\left[1-w\left(q_{k}\right)\right] \mathcal{I}_{k}^{(0)}+w\left(q_{k}\right)
$$

and replace $I_{k}$ in Eq. A5 and Eq. A6 with this newly defined function $\mathcal{I}_{k}^{*}$, while we keep $\mathcal{I}_{k}$, which appears in the equation of motion as it is (see Sec. III in Ref. [41]). The difference between $\mathcal{I}_{k}^{*}$ and $\mathcal{I}_{k}$ is only that the first term of Eq. A1 is multiplied by $w\left(q_{k}\right)$ so that $\mathcal{I}_{k}^{*} \sim 0$ when the nucleon $k$ is located inside of a fragment, whereas $\mathcal{I}_{k}^{*} \sim \mathcal{I}_{k}$ when the nucleon $k$ has only a few neighboring nucleons. In addition to this modification, we change the criteria to choose the nucleons that are used to measure temperature of the system. It has been shown that, to calculate the temperature of the system correctly, it is necessary to choose the subsystem consisting of the nucleons with negligible quantum effects among them based on only the nucleon spatial coordinates without using momentum variables (see Appendix B in Ref. [41]). For this purpose, there was a condition that the nucleons that are used to measure the temperature are chosen not to have more than one other nucleon within a distance of $3 \mathrm{fm}$ in Ref. [41]. We replace this condition with $\left\{k ; w\left(q_{k}\right) \geq 0.9\right\}$, which has similar meaning to the aforementioned condition and guarantees that the difference between $\mathcal{I}_{k}^{*}$ and $\mathcal{I}_{k}$ for these nucleons is $1 \%$ at 
most. This replacement is justified by the study that the measured temperatures are independent of the choice of nucleons utilized to measure the temperature as long as necessary conditions are satisfied (see Sec. VC in Ref. [41]).
[1] C. P. Montoya et al., Phys. Rev. Lett. 73, 3070 (1994).

[2] J. Lukasik et al., Phys. Rev. C 55, 1906 (1997), and references therein.

[3] T. Lefort et al., Nucl. Phys. A662, 397 (2000).

[4] W. Reisdorf et al., Nucl. Phys. A612, 493 (1997).

[5] J. Pochodzalla et al., Phys. Rev. Lett. 75, 1040 (1995).

[6] P. Danielewicz, R. Lacey, and W. G. Lynch, Science 298, 1592 (2002).

[7] C. Fuchs and H. H. Wolter, Eur. Phys. J. A 30, 5 (2006), and references therein.

[8] C. J. Horowitz, Eur. Phys. J. A 30, 303 (2006), and references therein.

[9] S. Das Gupta, A. Z. Mekjian, and M. B. Tsang, Adv. Nucl. Phys. 26, 89 (2001).

[10] M. D’Agostino et al., Nucl. Phys. A650, 329 (1999).

[11] M. D'Agostino et al., Phys. Lett. B473, 219 (2000).

[12] J. B. Natowitz et al., Phys. Rev. C 65, 034618 (2002).

[13] J. B. Elliott et al., Phys. Rev. Lett. 88, 042701 (2002).

[14] W. Trautmann, Nucl.Phys. A752, 407 (2005), and references therein.

[15] Ph. Chomaz and F. Gulminelli, Nucl. Phys. A647, 153 (1999).

[16] H. S. Xu et al., Phys. Rev. Lett. 85, 716 (2000).

[17] M. B. Tsang et al., Phys. Rev. C 64, 054615 (2001).

[18] D. H. E. Gross, Phys. Rep. 279, 120 (1997).

[19] J. Randrup and S. E. Koonin, Nucl. Phys. A471, 355 (1987).

[20] J. P. Bondorf, A. S. Botvina, A. S. Iljinov, I. N. Mishustin, and K. Sneppen, Phys. Rep. 257, 133 (1995).

[21] M. D’Agostino et al., Phys. Lett. B371, 175 (1996).

[22] A. S. Botvina and I. N. Mishustin, Eur. Phys. J. A 30, 121 (2006), and references therein.

[23] R. Wada et al., Phys. Lett. B422, 6 (1998).

[24] N. Marie et al., Phys. Lett. B391, 15 (1997).

[25] P. Désesquelles et al., Nucl. Phys. A633, 547 (1998).

[26] W. Reisdorf and H. G. Ritter, Annu. Rev. Nucl. Part. Sci. 47, 663 (1997).

[27] A. Andronic, J. Lukasik, W. Reisdorf, and W. Trautmann, Eur. Phys. J. A 30, 31 (2006), and references therein.

[28] A. Ono, H. Horiuchi, T. Maruyama, and A. Ohnishi, Phys. Rev. Lett. 68, 2898 (1992).

[29] A. Ono, H. Horiuchi, T. Maruyama, and A. Ohnishi, Prog. Theor. Phys. 87, 1185 (1992).

[30] A. Ono and H. Horiuchi, Prog. Part. Nucl. Phys. 53, 501 (2004).

[31] A. Ono, Phys. Rev. C 59, 853 (1999).

[32] A. Ono, S. Hudan, A. Chbihi, and J. D. Frankland, Phys. Rev. C 66, 014603 (2002).
[33] R. Wada et al., Phys. Rev. C 62, 034601 (2000).

[34] R. Wada et al., Phys. Rev. C 69, 044610 (2004).

[35] S. Hudan, R. T. de Souza, and A. Ono, Phys. Rev. C 73, 054602 (2006).

[36] A. Ono and H. Horiuchi, Phys. Rev. C 53, 2958 (1996).

[37] A. Ohnishi and J. Randrup, Phys. Rev. Lett. 75, 596 (1995).

[38] A. Ohnishi and J. Randrup, Ann. Phys. (N. Y.) 253, 279 (1997).

[39] A. Ono and H. Horiuchi, Phys. Rev. C 53, 845 (1996).

[40] A. Ono and H. Horiuchi, Phys. Rev. C 53, 2341 (1996).

[41] T. Furuta and A. Ono, Phys. Rev. C 74, 014612 (2006).

[42] D. H. E. Gross and E. V. Votyakov, Eur. Phys. J. B 15, 115 (2000).

[43] D. H. E. Gross, Phys. Chem. Chem. Phys. 4, 863 (2002).

[44] A. H. Raduta, M. Colonna, V. Baran, and M. Di Toro, Phys. Rev. C 74, 034604 (2006).

[45] A. H. Raduta, M. Colonna, and M. Di Toro, Phys. Rev. C 76, 024602 (2007).

[46] M. Colonna, G. Fabbri, M. Di Toro, F. Matera, and H. H. Wolter, Nucl. Phys. A742, 337 (2004).

[47] A. H. Raduta and A. R. Raduta, Phys. Rev. C 65, 054610 (2002).

[48] A. Ono, H. Horiuchi, and T. Maruyama, Phys. Rev. C 48, 2946 (1993).

[49] Y. Kanada-En'yo, Phys. Rev. C 71, 014310 (2005), and references therein.

[50] J. Dechargé and D. Gogny, Phys. Rev. C 21, 1568 (1980).

[51] K. Hagel et al., Phys. Rev. C 50, 2017 (1994).

[52] T. Maruyama, A. Ono, A. Ohnishi, and H. Horiuchi, Prog. Theor. Phys. 87, 1367 (1992).

[53] A. Ono, P. Danielewicz, W. A. Friedman, W. G. Lynch, and M. B. Tsang, Phys. Rev. C 70, 041604(R) (2004).

[54] S. Albergo, S. Costa, E. Costanzo, and A. Rubbino, Nuovo Cimento A 89, 1 (1985).

[55] W. P. Tan, S. R. Souza, R. J. Charity, R. Donangelo, W. G. Lynch, and M. B. Tsang, Phys. Rev. C 68, 034609 (2003).

[56] M. Di Toro, A. Olmi, and R. Roy, Eur. Phys. J. A 30, 65 (2006), and references therein.

[57] M. J. Ison, F. Gulminelli, and C. O. Dorso, Phys. Rev. E 76, 051120 (2007).

[58] F. Gulminelli and Ph. Chomaz, Nucl. Phys. A734, 581 (2004).

[59] M. B. Tsang et al., Phys. Rev. Lett. 92, 062701 (2004).

[60] Lie-Wen Chen, Che Ming Ko, and Bao-An Li, Phys. Rev. Lett. 94, 032701 (2005). 